\title{
Brain-scan ethics come under the spotlight
}

\section{Erika Check, Washington}

Suppose you're a neuroscientist studying human depression. In one study, you take pictures of depressed children's brains using a highly accurate imaging technique. Examining the data one day, you discover that one of your subjects is missing his entire parietal lobe, a crucial area for interpreting visual information. What do you do next?

Most scientists would refer the image to a physician, but this poses risks for both researchers and their subjects. Many scientists are unsure how to handle the legal, ethical and practical questions of accidental findings that could have implications for their subjects' health. These 'incidental findings' occur in many areas of biology, but cause particular problems in brain imaging.

The techniques used to study the brain, such as functional magnetic resonance imaging, are extremely powerful and are used more and more. And incidental findings are common. In a study released last October, $82 \%$ of brain-imaging researchers said they had turned up such findings, and $2-8 \%$ of research subjects have "clinically significant" findings, such as tumours, malformations or serious disease (J. Illes et al. J. Magn. Reson. Imag. 20, 743-747; 2004).

But handling incidental findings is complicated, especially in the United States, and procedures for dealing with them vary from institute to institute."We are uncovering increasing rates of incidental findings and discovering that there is no uniform protocol for dealing with them," says Judy Illes, an imaging researcher who directs the programme in neuroethics at the Stanford Center for Biomedical Ethics in California.

On 6 and 7 January, Illes led a workshop at the National Institutes of Health (NIH) in Bethesda, Maryland, to fill what one participant described as"a policy vacuum" on the question. About 50 scientists, physicians, lawyers and ethicists attended and agreed that every researcher should plan to cope with incidental findings before beginning brain-imaging studies.

Most felt that researchers should be allowed to draw up their own plans for dealing with these findings, but many were uncomfortable with the idea that basic researchers should ever be expected to spot medical information in non-clinical studies.

"It's changing the responsibility of a principal investigator, who has no medical training, to that of someone who is diagnosing a disease," said Steven Grant, a neuroscientist at the National Institute on Drug Abuse,

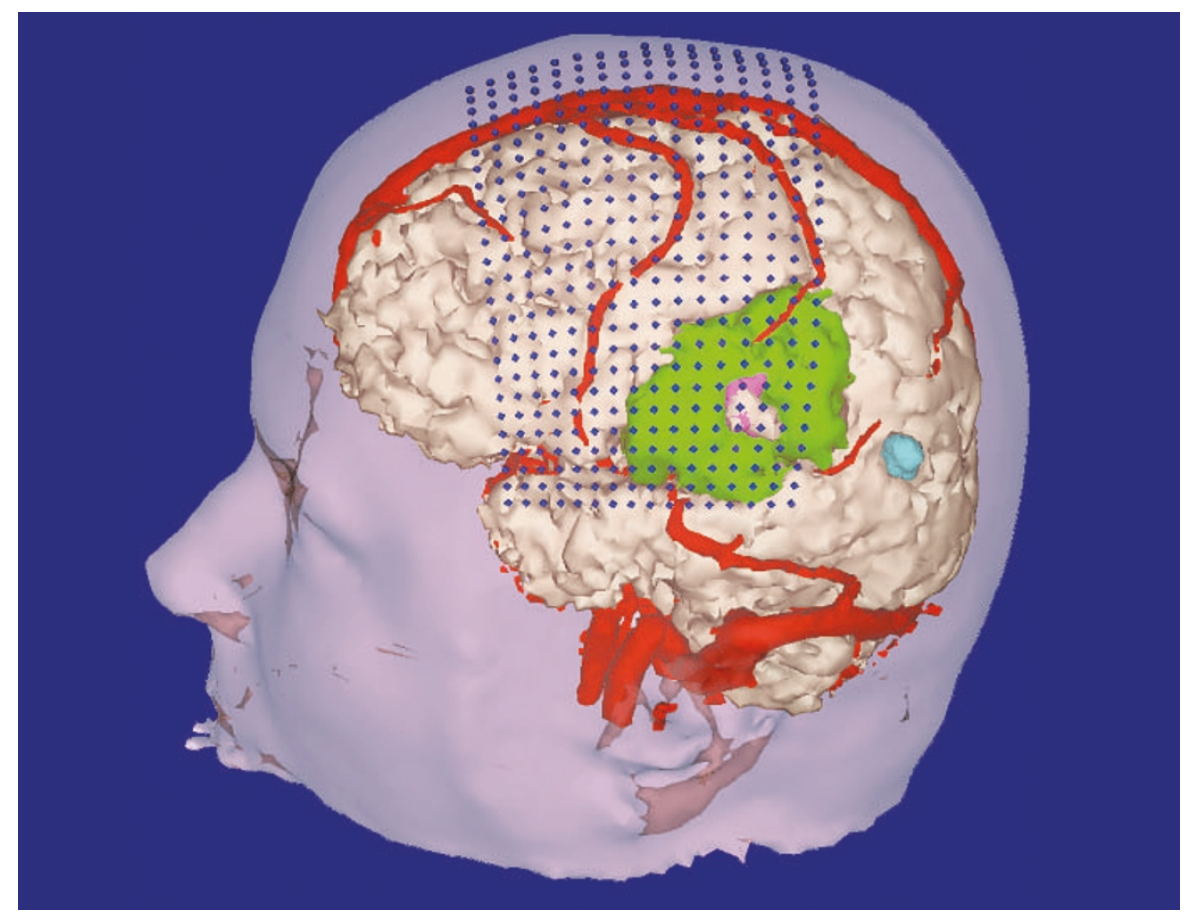

Three-dimensional brain scans, such as this magnetic resonance image, can reveal nasty surprises.

based in Bethesda, Maryland. "That changes the relationship."

For some, the best way to solve the problem is to ask a trained medical professional to examine every scan in every brain-imaging study. David Yousem, a neuroradiologist at Johns Hopkins University in Baltimore, Maryland, supports this idea: "It's about an ethical obligation to the person you recruited to the study to inform them of a medical abnormality," he said.

In the study by Illes et al., $23 \%$ of researchers surveyed followed this policy. The NIH goes one step further by requiring its on-campus investigators to perform a clinical scan of every research subject, in addition to any research scans. But workshop participants agreed that this was not a good idea because it can expose people to unnecessary risks from extra procedures.

Most researchers opt instead to send a radiologist only the brain scans that look obviously suspicious, such as the scan missing an entire parietal lobe. Illes et al. found that 53\% of researchers they surveyed did this.

But a case-by-case referral to a radiologist raises a host of other problems. An incidental medical finding may affect a subject's ability to buy health insurance, for example, and findings that turn out to be medically insignificant can still cause tremendous stress for research subjects and their families.
Walter Schneider, a psychologist at the University of Pittsburgh, Pennsylvania, said that a participant in one of his research studies decided not to go to graduate school because of a perceived defect in a brain scan.

For some basic researchers, the scariest aspect of entering the medical realm is the threat of lawsuits. If they accept any responsibility for detecting medical information, researchers fear, they will be legally accountable for diagnosing brain problems that are completely unrelated to their studies. "One of the things that should be taken into consideration is the clinical research that won't be done because of the cost, and because the liability makes people nervous," said Alan Evans, a neurologist at McGill University in Montreal, Canada.

Lawyer Susan Wolf of the University of Minnesota, Minneapolis, pointed out that US courts are becoming increasingly likely to hold researchers responsible for their subjects' welfare. In Maryland, for example, a state appeals court decided in 2001 that researchers studying lead abatement procedures in Baltimore should have warned some children in the study that they had dangerous levels of lead in their blood. "We are already out of the era in which researchers had no legal responsibilities to their subjects,"Wolf said.

Illes had hoped to agree general guidelines at the January meeting. But after heated debate, the group could not even decide whether every study should refer suspicious findings to a medical professional. Illes hopes to convene additional meetings to forge agreement on this point, among others. 